\title{
Acupuncture and Moxibustion Theories of Zhang $\mathrm{Ji}^{*}$
}

\author{
Yong Chen ${ }^{1,2 \#}$, Yinmin Le ${ }^{1}$, Jia Wei ${ }^{1}$ \\ ${ }^{1}$ Jiangxi University of Traditional Chinese Medicine, Nanchang, China \\ ${ }^{2}$ Fuda Cancer Hospital, Guangzhou, China \\ Email: "shanggongchenyong@163.com
}

Received July 13, 2012; revised August 14, 2012; accepted August 20, 2012

\begin{abstract}
This paper introduces the clinical experiences on acupuncture and moxibustion of Zhang Zhongjing, who was regarded as "medical Saint" of Traditional Chinese Medicine. He gave indications for acupuncture and moxibustion, developed robbing fire or inversing fire acupuncture principles to treat febrile diseases. His theories on acupuncture and moxibustion are precious and could be reference in clinical practice.
\end{abstract}

Keywords: Zhang Zhongjing; Treaties on Febrile and Miscellaneous Diseases; Golden Chamber Synopsis; Indication; Theory; Acupuncture Methods

Zhang Ji (about AD 150-219), also known as Zhang Zhongjing, was born in the Nieyang, Nangyang (known as Denxian, Henan province today) in the late age of East Han dynasty. The world honors him as Medical Saint. His works include 伤寒杂病论 (Treaties on Febrile and Miscellaneous Diseases), 金度要略 (Synopsis of Golden Chamber). Even today, the two books are the compulsory classical courses in Traditional Chinese Medical Universities. His advocation on treatments according to syndromes differentiation by six channels and eight principles has great influences to the development of Chinese medicine afterwards.

On clinical, Zhang Zhongjing mainly treated diseases with formular medicine, however, there are dozens of terms related with acupuncture and moxibustion theropy. The acupoints mentioned in his books includ Fengfu (GV 16), Fengchi (GB 20), Qimen (LR 14), Dazhui (GV 14), Feishu (BL 13), Ganshu(BL 18), Laogong (PC 8), Guanyuan(RN 4) etc. The equipments and methods for acupuncture and moxibustion include needle (needling), moxa (moxibustion), fumigation, burning and warming acupuncture ect. are mentioned. Various diseases or symptoms were treated by not a few therapeutic methods of acupuncture and moxibustion, which have practicle influences to guidance of bedside acupuncture and moxibustion.

\section{Theory of “当刺” (Need Acupuncture), “可多” (Can Moxibustion)}

The theory of "need acupuncture" often appeared in

\footnotetext{
${ }^{*}$ This paper is translated from Acupuncture and Moxibustion Schools of Ancient Famous Practitioners, which is edited by Wei Jia, Gao Xiyan. The work is sponsored by People's Healthy Publish House, China.

"Corresponding author.
}

Zhongjing's books. Such as, the $148^{\text {th }}$ term from Treaties on Febrile and Miscellanous Diseases and the $22^{\text {nd }}$ term from Synopsis of Golden Chamber (For each cited in the following passage, if not specifically indicated (the $x x^{\text {th }}$ term), the quotation is from Treaties on Febrile and Miscellanous Diseases, if the quotation is from Golden Chamber Synopsis, it will be specified in the text) reads: A woman was attacked by wind, fever, aversion to cold. Her menstruation was due and lasted for 7 to 8 days. After the fever was gone, her pulse was slow, cold body, chest fullness and stagnation sensation, delirious speech. These are the manifestations of heat entering blood chamber, acupuncture on Qimen is needed. The $221^{\text {st }}$ term reads: When Yangming of a patient is attacked, the hematochezia and delirious speech suggested the heat entering blood chamber, if the patient had sweating head, need acupuncture on Qimen. The $147^{\text {th }}$ term reads: If Taiyang and Shaoyang meridians of a patient is attacked at the same time, manifestations are headache and neck stiffness, or vertigo, sometimes feel stagnation of chest, stiffness associated with local rigidity in epigastric region, needs to acupuncture on Dazhui, Feishu, Ganshu. Be cautious that diaphoresis is not proper for the patient. If diaphoresis was applied to the patient, he or she would have delirious speech for 5 days, wiry pulse. It needs acupuncture on Qimen to slove the problem.

Sometimes, Zhongjing used the terminologies of “可 刺”'(can acupuncture), or “宜针”(acupuncture is appropriate). Such as, the $308^{\text {th }}$ term reads: If Shaoyin is attacked, the patient has dysentery, bloody and purulent stool, can be treated by acupuncture. The $6^{\text {th }}$ term from Golden Chamber Synopsis reads: For blood bi syndrome 
(blood-arthragia)... using acupuncture to guide the Yang qi is appropriate. Sometimes, only "needling” is the word. Such as, the $112^{\text {nd }}$ term describes the fever with aversion to wind, thirsty, abdominal distension, spontaneous perspiration... needle the Qimen. The $111^{\text {st }}$ term reads: Fever with abdominal distension, delirious speech, floating and tight cun pulse... needle the Qimen. The $19^{\text {th }}$ term from Golden Chamber Synopsis reads: Fujue (means the rigidity of foot, especially the flex function) [1], the patient can move forwards but hardly stop (by her/himself), needling 2 cun into the calf.

Generally, as to apply needles for treatment, “当刺”, (need acupuncture), “可刺” (can acupuncture), “宜钎” (acupuncture is appropriate), “刺” (needling) have the same meaning. The terms from the two books discussed 6 syndromes indicated by acupuncture, which are: Firstly, heat symptom of Sanyang (Triyang: Taiyang, Shaoyang, Yangming). Secondly, heat entering blood chamber, which means during the menstruation, before or after menstruation, the woman affected by external evils, heat evil lands in blood chamber (uterus). The manifestations include fever and aversion to cold, distension of chest and abdomen, delirious speech. Thirdly, stiffness and pain of neck, vertigo, and acupuncture should be the first choice with satisfied outcome. Fourthly, bloody and purulent stool, include the so called dysentery afterwards. Acupuncture proved to be very efficient for dysentery patients. Fifthly, blood-bi (blood-arthragia) [2], which mainly manifested as numbness of limbs. Acupuncture therapy is indicated for this type of diseases obviously. Sixthly, Fujue symptoms manifested as rigidity of acrotarsium, hard to walk. The clinical observation proved acupuncture has significant effectiveness for this type of diseases also.

Speaking of moxibustion, Zhongjing has the theories like “可炎” (can moxibustion), “当炎” (need moxibustion), “炎之” (apply moxibustion), “熏之” (fumigate). The $349^{\text {th }}$ term reads: Fever with rapid pulse, cold extremities, can use moxibustion (to treat). The $304^{\text {th }}$ term reads: Shaoyin disease, if lasts for 1-2 days, normal sense of mouth, chilly sensation in the back, the patient needs moxibustion treatment. The $17^{\text {th }}$ term from Golden Chamber Synopsis and the $361^{\text {st }}$ term reads: For diarrhoea, cold extremities, pulselessness patients, apply moxibustion... The $325^{\text {th }}$ term reads: Shaoyin disease, diarrhoea, microastringent pulse, vomiting, sweating, the patient must change clothes frequently. Otherwise, apply moxibustion to warm Shaoyin.

Therefore, the indication range of moxibustion including: Cold extremities, aversion to cold, diarrhoea, weak pulse ect manifested in Sanyin (Triyin, which is Taiyin, Shaoyin, Queyin) diseases. It suggests that moxibustion treatment has function of restoring Yang saving inverse, which has differences compared using acupuncture to treat Sanyang diseases mentioned above. How- ever, it is not absolutely impossible to treat Yang syndrome with moxibustion whilst acupuncture to Yin syndrome. Such as, in the $487^{\text {th }}$ term applying fumigation to bi-yang disease; in the $308^{\text {th }}$ term using acupuncture to treat diarrhoea of Shaoyin disease. We may infer that at bed side of patients, Zhongjing stressed the treatments according to syndromes differentiation.

\section{Theory of “火逆” (Inversing Fire), “火劫” (Robbing Fire)}

Zhang zhongjing, who was famous for his treatment of febrile diseases with decoctions, also persisting to the principles of treating cold diseases with heat medicine, while treating heat diseases with cold medicine (warm the cold, cool the heat). He thought the heat from the moxa fire same as the heat from herbals, and has vigilance on moxibustion treatment. He mentioned “火逆” (inversing fire), “火却” (robbing fire), “火邪” (fire evil), “火动” (fire stirring), “火攻” (fire attack), “火盛” (surpassing fire), “被火” (burning/being on fire) ect. repeatedly, to raise attentions of following practitioners. The $119^{\text {th }}$ term reads: Caused by fire evil, the patient manifested as irratation, reflex sensation. The $120^{\text {th }}$ term describes, if the disease is located in external and treated mistakenly by moxibustion, fire would cause the disease severer, leads to inversing fire syndrome which is severe bi (arthralgia) of lower back. The $115^{\text {th }}$ term describes if the fever with floating pulse was treated by moxibustion, may leads to the side effects of Yang depletion or mania. The $284^{\text {th }}$ term describes the diarrhea, cough, and delirious speech of shaoyin disease is caused by robbing fire. The $117^{\text {th }}$ term reads: Taiyang disease, treated by fumigation, no sweating, would cause the restlessness of the patient..., which is named fire evil. The $2^{\text {nd }}$ term from Golden Chamber Synopsis reads: The patient with irritation and pain caused by dampness, needs diaphoresis, while attacking fire (moxibustion) is not appropriate. The $118^{\text {th }}$ term reads: If pulse is floating, and the patient has extremely heat, this is excess. The excess needs to be treated by $\mathrm{xu}$ (difficiency). But if treated by moxibustion, the stirring fire can leads to dryness of throat, and hemoptysis. The $205^{\text {th }}$ term reads: If Yangming is been burning by fire... leads to yellowish complexion. The $6^{\text {th }}$ term describes: Taiyang disease, manifests as fever, thirsty, no aversion to cold is belong to warm disease. If treated by fire, could leads to epilepsy, clonic convulsion. One time of inversing fire may cause the delay of recovery, repeatedly inversing fire may shorten patient's survival time. The $205^{\text {th }}$ term reads: If pulse is faint and rapid, no moxibustion. The fire seems mini, but have strength of inside attack, can burning bones and tendons, losing of blood. The $2^{\text {nd }}$ term of Golden Chamber Synopsis reads: convulsion and skin ulcer caused by moxibustion are hard to cure. The $25^{\text {nd }}$ term of Golden Chamber Synopsis 
reads: Drunk patients should not applied moxibustion in abdomina nor back. Otherwise the stagnation of intestine happens.

Above all, Zhongjing suggested the diseases and symptoms that need avoid of moxibustion include: Faint and rapid pulse, floating pulse, Taiyang syndrome, Yangmin syndrome, dampness causing the irritation and pain of the patient, heat syndrome (over excess of Yang or Yin differency), thirsty, excess, warm diseases ect., all of these regarded as Yang symptoms. He think the mismoxibustion may cause the side effects of irritation, reflex sensation, heavy bi in lower back, epilepsy, clonic convulsion, delirious speech, dry throat and hemoptysis, yellowish complexion... even leads to the disastrous effect like shortening life expectancy.

\section{Theory of Warming or Burning Acupuncture}

The theory of warming acupuncture and burning acupuncture is originated from Neijing (Internal Canon of Medicine). The $128^{\text {th }}$ term describes that if fever of Taiyang disease treated by warming acupuncture would cause convulsion in the patient. The $226^{\text {th }}$ term describes: If Yangming disease manifest as floating and tight pulse, dry throat, no aversion to cold but heat, then treated by warming acupuncture would cause apprehensiveness, irratation, and insomnia. The $267^{\text {th }}$ term describes: Taiyang disease transfers to Shaoyang, and manifests as hypochondrium fullness and rigidity, retching and cannot eating, alternating of chills and fever, methods of diaphoresis, emetic therapy and purgation are done, if add warming acupuncture would lead to delirious speech. The $2^{\text {nd }}$ term of Golden Chamber Synopsis describes: Taiyang attacked by summer heat could manifests as fever and aversion to cold, heavy body and general pain... diaphoresis may worsen the aversion to cold, warming acupuncture may worsen the fever. The $29^{\text {th }}$ term describes: If fever with floating pulse... treated by diaphoresis and burning acupuncture can cause Yang depletion to the patient. The $121^{\text {st }}$ term describes: Burning acupuncture may cause sweating of the location, if the acupuncture region attacked by cold may grow a red nut-like mass. One moxacone of moxibustion may be applied to handle the problem. The $122^{\text {nd }}$ term describes: Burning acupuncture may cause irritation of the patient. The $158^{\text {th }}$ term describes: After treat Taiyang disease with diaphoresis, both Yin and Yang qi are exhausted, if add burning acupuncture, which may cause irritation of the patient.

Due to the burning acupuncture and worming acupuncture needs fire during the process, Zhongjing regarded them as similar to the inversing fire or robbing fire in moxibustion. Burning and warming acupuncture are special treatment methods, which combine acupuncture and moxibustion together, and still in widely using by far. The terminologies in Zhongjing's books are among the earliest recordations with relatively high historic value. Pitifully, Zhongjing didn't record the detail manipulation of the technicals.

\section{Combination of Acupuncture and Drugs}

At bed side, when give treatments, Zhongjing can combine acupuncture and drugs, fully use them advantages, which is estimable deeds. Chapter $22^{\text {nd }}$ pulse symptoms and treatments of woman miscellaneous diseases in Golden Chamber Synopsis reads: ... of thirty-six diseases, with thousands of changes, practitioners need to feel the Yin and Yang of the pulses... combine acupuncture and drugs can cure severe diseases... Nowadays clinical dates show combination of acupuncture and drugs can bring better effectiveness for obstetrical and gynecological diseases like menstruation disorder, pelvic infection, mastitis, infertility, abnormal fetal position, dystocia ect. The $24^{\text {th }}$ term reads: A Taiyang disease patient, treated by Guizhi decoction at first, more irritable without relieve. Add acupuncture on Fengchi, Fengfu, the patient recoered soon. The effectiveness of treating influenza (external syndrome of Taiyang) has been widely accepted today. The $4^{\text {th }}$ term from Golden Chamber Synopsis reads: malaria... could be treated with diaphoresis, acupuncture and moxibustion. There are amounts of researches on acupuncture for malaria. For some diseases, acupuncture may have less effect, add drugs to make up the defect. For example, the $234^{\text {th }}$ term discusses the wind attacks the Yangming, needling has less effect, the Xiaochaihu decoction can be helpful [3].

Besides the theories discussed above, Zhongjing also advocates the principles of prevention is better than cure, and treating diseases in early stage. The first part of Golden Chamber Synopsis states: "the pathogenic evils can enter the zang-fu organs by Jingluo (meridians and channels)", "if one pays attention to health, he/she won't let the evil wind interfere the Jingluo. If the evil attacks a Jingluo, he/she will seek therapies before it enter into zang-fu organs”. Can apply “daoyin (physical and breathing exercise)... acupuncture and moxibustion..." to restrain the development of the disease. The $8^{\text {th }}$ term states: When a disease is transferring from Taiyang to Yangming, "acupuncture on Yangming, can stop the transferring, and cure the disease”, which indicates acupuncture can prevent the deterioration of diseases, and accelerates the healing process. These viewpoints are of great significances.

\section{REFERENCES}

[1] Q. X. Zeng, “Analysis of 'Fujue’ Term of Golden Chamber Synopsis,” Chinese Journal of Basic Medicine, Vol. 7, No. 8, 2001, pp. 568-569. 
[2] J. Z. Zhou and X. H. Liu, "Blood-Bi and Cardiovascular Diseases," Chinese Journal of Integrative Medicine on Cardio/ Cerebrovascular Disease, Vol. 7, No. 7, 2007, pp. 612-613.
[3] Z. J. Zhang, "Synopsis of Golden Chamber," Shanghai Press of Classics, Shanghai, 2010, pp. 152-154. 